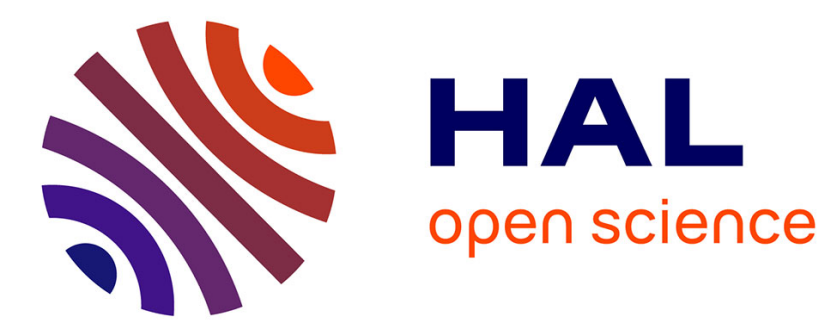

\title{
Global a priori convergence theory for reduced-basis approximations of single-parameter symmetric coercive elliptic partial differential equations
}

\author{
Yvon Maday, Anthony T. Patera, Gabriel Turinici
}

\section{- To cite this version:}

Yvon Maday, Anthony T. Patera, Gabriel Turinici. Global a priori convergence theory for reducedbasis approximations of single-parameter symmetric coercive elliptic partial differential equations. Comptes rendus de l'Académie des sciences. Série I, Mathématique, 2002, 335 (3), pp.289-294. 10.1016/S1631-073X(02)02466-4 . hal-00798389

\section{HAL Id: hal-00798389 \\ https://hal.science/hal-00798389}

Submitted on 11 Mar 2013

HAL is a multi-disciplinary open access archive for the deposit and dissemination of scientific research documents, whether they are published or not. The documents may come from teaching and research institutions in France or abroad, or from public or private research centers.
L'archive ouverte pluridisciplinaire HAL, est destinée au dépôt et à la diffusion de documents scientifiques de niveau recherche, publiés ou non, émanant des établissements d'enseignement et de recherche français ou étrangers, des laboratoires publics ou privés. 


\title{
Global a Priori Convergence Theory for Reduced-Basis Approximations of Single-Parameter Symmetric Coercive Elliptic Partial Differential Equations
}

\author{
Yvon MADAY ${ }^{a}$, Anthony T. PATERA ${ }^{b}$, G. TURINICI $^{c}$ \\ a Analyse numérique, Université Paris VI, 4, place Jussieu, 75252 Paris cedex 05, France \\ b Department of Mechanical Engineering, M.I.T., 77 Mass. Ave., Cambridge, MA, 02139, USA \\ c INRIA Rocquencourt M3N, B.P. 105, 78153 Le Chesnay Cedex France
}

\begin{abstract}
We consider "Lagrangian" reduced-basis methods for single-parameter symmetric coercive elliptic partial differential equations. We show that, for a logarithmic-(quasi-)uniform distribution of sample points, the reduced-basis approximation converges exponentially to the exact solution uniformly in parameter space. Furthermore, the convergence rate depends only weakly on the continuity-coercivity ratio of the operator: thus very low-dimensional approximations yield accurate solutions even for very wide parametric ranges. Numerical tests (reported elsewhere) corroborate the theoretical predictions.

Résultats globaux a priori pour l'approximation d'équations aux dérivées partielles coercives symétriques elliptiques dépendant d'un paramètre
Résumé. On considère des méthodes de bases réduites de type Lagrange pour des équations aux dérivées partielles coercives symétriques elliptiques et dépendant d'un paramètre. On montre que, pour une répartition logarithmiquement quasi uniforme des points d'échan- tillonage, l'approximation en base réduite converge de façon exponentielle vers la solution exacte uniformément par rapport au paramètre. De plus la convergence ne dépend que faiblement du rapport entre les coefficients de coercivité et de continuité de l'opérateur: ainsi une approximation de très basse dimension procure une solution très précise même dans le cas d'un large eventail de paramètres. Des test numériques (reportés par ailleurs) corroborent ces prédictions numériques

Version française abrégée Dans un espace de Hilbert $H$, muni du produit scalaire $(\cdot, \cdot)_{Y}$ et de la norme $\|\cdot\|_{Y}$ on se pose le problème de trouver $u \in Y$ vérifiant (1) où la forme bilinéaire $a: Y \times Y \times \mathcal{D} \rightarrow \mathbb{R}$ dépend d'un paramètre $\mu \in \mathcal{D} \equiv\left[0, \mu_{\text {max }}\right]$. Sous des conditions classiques de continuité et de coercivité de a ce problème possède une solution unique. La méthode de base réduite consiste alors à choisir un entier $N$ et un jeux de paramètres $S_{N}=\left\{\alpha_{1}, \ldots, \alpha_{N}\right\}$ pour lesquels, de façon préalable, on calcule - le plus exactement possible - les solutions associées $u\left(\alpha_{k}\right), k=1, \ldots, N$.Puis on résout le système $(2)$ où $W_{N}=\operatorname{Vect}\left\{u\left(\alpha_{k}\right), k=1, \ldots, N\right\}$. On analyse dans cette note le cas d'un problème dépendant d'un seul paramètre du type (3) où $a_{0}: Y \times Y \rightarrow \mathbb{R}$ et $a_{1}: Y \times Y \rightarrow \mathbb{R}$ sont continues, symétriques, semi positives et de plus où $a_{0}$ est coercive induisant une norme \|\|$\cdot \|\left.\right|^{2}=a_{0}(\cdot, \cdot)$ équivalente à celle de $Y$. Des exemples de problèmes entrant dans ce cadre sont présentés, analysés et simulés sur base réduite dans [12]. Plus particulièrement nous montrons ici que la convergence de cette méthode en base réduite est une fonction exponentiellement décroissante en le cardinal de $W_{N}$, et ce uniformément par rapport au 
paramètre. En particulier on a la borne suivante entre la solution exacte $u(\mu)$ et son approximation $u_{N}(\mu)$ : il existe un entier $N_{\text {crit }}$ tel que pour tout $N \geq N_{\text {crit }}$, on a (19) avec une constante $c$ ne dépendant que des conditions d'ellipticité de $a_{0}$ et de $\mu_{\max }$.

La démonstration de ce résultat repose d'une part sur le lemme classique de Cea rappelé en (10) et une estimation a priori de la meilleure approximation donnée dans le lemme 2.

Il convient de noter que l'analyse de la meilleure approximation fait ici intervenir une approximation polynomiale de la solution, mais cette approximation polynomiale est proposée après un changement de variable approprié $\left(\mu=e^{\tilde{\mu}}-\gamma^{-1}\right)$. Le point qui doit être noté est que la méthode de Galerkin propose naturellement une approximation dans $W_{N}$ qui est (à une constante multiplicative près) aussi bonne que cette approximation polynomiale en une variable à définir. Ceci donne une supériorité et un caractère général à l'approche variationelle par rapport à une "simple" interpolation puisque aucune connaissance a priori de la forme de la solution en son paramètre n'est à connaître.

L'analyse faite ici suggère une répartition logarithmique du jeux de paramètres qui donne en effet de meilleurs résultats dans les applications comme celà est reporté dans [15]. On renvoit aussi à [12] pour plus de détails sur la mise en oeuvre et les applications.

\section{Introduction}

Let $Y$ be an Hilbert space with inner product and norm $(\cdot, \cdot)_{Y}$ and $\|\cdot\|_{Y}=(\cdot, \cdot)_{Y}^{1 / 2}$, respectively. Consider a parametrized "bilinear" form $a: Y \times Y \times \mathcal{D} \rightarrow \mathbb{R}$, where $\mathcal{D} \equiv\left[0, \mu_{\max }\right]$, and a bounded linear form $f: Y \rightarrow \mathbb{R}$. We introduce the problem to be solved: Given $\mu \in \mathcal{D}$, find $u \in Y$ such that

$$
a(u(\mu), v ; \mu)=f(v), \quad \forall v \in Y .
$$

Under natural conditions on the bilinear form a (e.g. continuity and coercivity) it is readily shown that this problem admits a unique solution.

We introduce an approximation index $N$, the parameter sample $S_{N}=\left\{\alpha_{1}, \ldots, \alpha_{N}\right\}$, and the solutions $u\left(\alpha_{k}\right), k=1, \ldots, N$, of problem (1) for this set of parameters. We next define the reducedbasis approximation space $W_{N}=\operatorname{span}\left\{u\left(\alpha_{k}\right), k=1, \ldots, N\right\}$. Our reduced-basis approximation is then: Given $\mu \in \mathcal{D}$, find $u_{N}(\mu) \in W_{N}$ such that

$$
a\left(u_{N}(\mu), v ; \mu\right)=f(v), \quad \forall v \in W_{N} .
$$

This discrete problem is well posed under the same former continuity and coercivity conditions.

The reduced-basis approach, as earlier developped, is typically local in parameter space in both practice and theory $[1,2,4,9,10,13]$. To wit, the $\alpha_{k}$ are chosen in the vicinity of a particular parameter point $\mu^{*}$ and the associated a priori convergence theory relies on asymptotic arguments in sufficiently small neighborhoods of $\mu^{*}$ [4]. In this note we present, for single-parameter symmetric coercive elliptic partial differential equations, a first theoretical a priori convergence result that demonstrates exponential convergence of reduced-basis approximations uniformly over an extended parameter domain. The proof requires, and thus suggests, a point distribution in parameter space which does, indeed, exhibit superior convergence properties in a variety of numerical tests [15]. We refer also to $[5,6,7,12]$ for further discussions of these results and related work and applications.

\section{Problem Formulation}

Let us define the parametrized "bilinear" form $a: Y \times Y \times \mathcal{D} \rightarrow \mathbb{R}$ as

$$
a(w, v ; \mu) \equiv a_{0}(w, v)+\mu a_{1}(w, v)
$$


where the bilinear forms $a_{0}: Y \times Y \rightarrow \mathbb{R}$ and $a_{1}: Y \times Y \rightarrow \mathbb{R}$ are continuous, symmetric and positive semi-definite; suppose moreover that $a_{0}$ is coercive, inducing a ( $Y$-equivalent) norm $\|\cdot\| \|^{2}=$ $a_{0}(\cdot, \cdot)$. It follows from our assumptions that there exists a real positive constant $\gamma_{1}$ such that

$$
0 \leq \frac{a_{1}(v, v)}{a_{0}(v, v)} \leq \gamma_{1}, \quad \forall v \in Y
$$

For these hypotheses, it is readily demonstrated that the problem (1) has a unique solution.

Many situations may be modeled by our rather simple problem statement (1), (3). For example, if we take $Y=H_{0}^{1}(\Omega)$ where $\Omega$ is a smooth bounded subdomain of $\mathbb{R}^{d=2}$, and set $a_{0}(w, v)=$ $\int_{\Omega} \nabla w \cdot \nabla v, a_{1}=\int_{\Omega} w v$, we model conduction in thin plates; here $\mu$ represents the convective heat transfer coefficient. Other choices of $a_{0}$ and $a_{1}$ can model variable rectilinear geometry, variable orthotropic properties, and variable Robin boundary conditions.

The space $Y$ is typically of infinite dimension so $u(\mu)$ is, in general, not exactly calculable. In order to construct our reduced-basis space $W_{N}$, we must therefore replace $u(\mu) \in Y$ by a "truth approximation" $u^{\mathcal{N}}(\mu) \in Y^{\mathcal{N}} \subset Y$, solution of the Galerkin approximation $a\left(u^{\mathcal{N}}(\mu), v ; \mu\right)=$ $f(v), \forall v \in Y^{\mathcal{N}}$. Here $Y^{\mathcal{N}}$, of finite (but typically very high) dimension $\mathcal{N}$, is a sufficiently rich approximation subspace such that $\left\|u(\mu)-u^{\mathcal{N}}(\mu)\right\| \|$ is sufficiently small for all $\mu$ in $\mathcal{D}$; for example, for $Y=H_{0}^{1}(\Omega)$ we know that, for any desired $\varepsilon>0$, we can indeed construct a finite-element approximation space, $Y^{\mathcal{N}(\varepsilon)}$, such that $\left\|u(\mu)-u^{\mathcal{N}(\varepsilon)}(\mu)\right\| \mid \leq \varepsilon$.

It shall prove convenient in what follows to introduce a generalized eigenvalue problem: Find $\left(\varphi_{i}^{\mathcal{N}} \in Y^{\mathcal{N}}, \lambda_{i}^{\mathcal{N}} \in \mathbb{R}\right), i=1, \ldots, \mathcal{N}$, satisfying $a_{1}\left(\varphi_{i}^{\mathcal{N}}, v\right)=\lambda_{i}^{\mathcal{N}} a_{0}\left(\varphi_{i}^{\mathcal{N}}, v\right), \forall v \in Y^{\mathcal{N}}$. We shall order the (perforce real, non-negative) eigenvalues as $0 \leq \lambda_{\mathcal{N}}^{\mathcal{N}} \leq \lambda_{\mathcal{N}-1}^{\mathcal{N}} \leq \cdots \leq \lambda_{1}^{\mathcal{N}} \leq \gamma_{1}$, where the last inequality follows directly from (4). We may choose our eigenfunctions such that

$$
a_{0}\left(\varphi_{i}^{\mathcal{N}}, \varphi_{j}^{\mathcal{N}}\right)=\delta_{i j}
$$

and hence $a_{1}\left(\varphi_{i}^{\mathcal{N}}, \varphi_{j}^{\mathcal{N}}\right)=\lambda_{i}^{\mathcal{N}} \delta_{i j}$, where $\delta_{i j}$ is the Kronecker-delta symbol; and such that $Y^{\mathcal{N}}$ can be expressed as $\operatorname{span}\left\{\varphi_{i}, i=1, \ldots, \mathcal{N}\right\}$. Note that, thanks to the finite dimension of our approximation space $Y^{\mathcal{N}}$, we preclude (the complications associated with) a continuous spectrum - and, as we shall see, at no loss in rigor.

We conclude this section by noting that, if we set $f_{i}^{\mathcal{N}}=f\left(\varphi_{i}^{\mathcal{N}}\right)$, then $u^{\mathcal{N}}(\mu)$ can be expressed as

$$
u^{\mathcal{N}}(\mu)=\sum_{i=1}^{\mathcal{N}} \frac{f_{i}^{\mathcal{N}} \varphi_{i}^{\mathcal{N}}}{1+\mu \lambda_{i}^{\mathcal{N}}}
$$

\section{A Priori Convergence Theory}

We propose here to choose the sample points $\alpha_{k}, k=1, \ldots, N$, log-equidistributed in $\mathcal{D}$, where $\delta_{N}=\ln \left(\gamma \mu_{\max }+1\right) / N$, and $\gamma$ is any finite upper bound for $\gamma_{1}{ }^{1}$. Here $\tilde{\delta}_{k N} / \delta_{N} \leq c^{*}, \quad \forall k, k=$ $1, \ldots, N$, and also $\sum_{\ell=1}^{N} \tilde{\delta}_{\ell N}=\ln \left(\gamma \mu_{\max }+1\right)$, where $c^{*}$ is a real positive constant.

Denote the reduced-basis approximation space as $W_{N}^{\mathcal{N}}=\operatorname{span}\left\{u^{\mathcal{N}}\left(\alpha_{k}\right), k=1, \ldots, N\right\}$. Although in general $\operatorname{dim}\left(W_{N}^{\mathcal{N}}\right) \leq N$, we can suppose that $\operatorname{dim}\left(W_{N}^{\mathcal{N}}\right)=N$ (otherwise we eliminate elements from $W_{N}^{\mathcal{N}}$ until it contains only linearly independent vectors). Then, the (reduced basis) problem is : Given $\mu \in \mathcal{D}$, find $u_{N}^{\mathcal{N}}(\mu) \in W_{N}^{\mathcal{N}}$ such that

$$
a\left(u_{N}^{\mathcal{N}}(\mu), v ; \mu\right)=f(v), \quad \forall v \in W_{N}^{\mathcal{N}}
$$

$\overline{1 \text { Note that } \gamma_{1}, \gamma \text {, and hence } S_{N}}$, are independent of $\mathcal{N}$. 
This problem admits a unique solution.

Our goal is to (sharply) bound $\left\|\mid u^{\mathcal{N}}(\mu)-u_{N}^{\mathcal{N}}(\mu)\right\| \|$, for all $\mu \in \mathcal{D}$, as a function of $N$ (and ultimately $\mathcal{N}$ as well). This error bound in the energy norm can be readily translated into error bounds on continuous-linear-functional outputs [12]; we do not consider this extension further here.

We shall need two standard results from the theory of Galerkin approximation of symmetric coercive problems [14]:

$$
\begin{gathered}
a\left(u^{\mathcal{N}}-u_{N}^{\mathcal{N}}, u^{\mathcal{N}}-u_{N}^{\mathcal{N}} ; \mu\right)=\inf _{w_{N}^{\mathcal{N}} \in W_{N}^{\mathcal{N}}} a\left(u^{\mathcal{N}}-w_{N}^{\mathcal{N}}, u^{\mathcal{N}}-w_{N}^{\mathcal{N}} ; \mu\right) ; \\
a\left(u^{\mathcal{N}}, u^{\mathcal{N}} ; \mu\right) \leq a(u, u ; \mu) .
\end{gathered}
$$

From the positive semidefiniteness of $a_{1},(3),(4)$ and (8) we can write

$$
\begin{aligned}
\left.\left\|u^{\mathcal{N}}(\mu)-u_{N}^{\mathcal{N}}(\mu)\right\|\right|^{2} & \leq a\left(u^{\mathcal{N}}(\mu)-u_{N}^{\mathcal{N}}(\mu), u^{\mathcal{N}}(\mu)-u_{N}^{\mathcal{N}}(\mu), \mu\right) \\
& \leq \inf _{w_{N}^{\mathcal{N}} \in W_{N}^{\mathcal{N}}} a\left(u^{\mathcal{N}}(\mu)-w_{N}^{\mathcal{N}}, u^{\mathcal{N}}(\mu)-w_{N}^{\mathcal{N}}, \mu\right) \\
& \leq\left.\left(1+\mu_{\max } \gamma_{1}\right) \inf _{w_{N}^{\mathcal{N}} \in W_{N}^{\mathcal{N}}}\left\|u^{\mathcal{N}}(\mu)-w_{N}^{\mathcal{N}}\right\|\right|^{2}, \quad \forall \mu \in \mathcal{D} .
\end{aligned}
$$

Also from the definition of the ||$|\cdot|||$ norm and the positive semidefiniteness of $a_{1},(3)$, (4) and (9), we obtain

$$
||\left|u ^ { \mathcal { N } } ( \mu ) \left\||| \leq\left(1+\mu_{\max } \gamma_{1}\right)^{1 / 2}|\|u(\mu) \mid\|, \quad \forall \mu \in \mathcal{D} .\right.\right.
$$

We first state a preparatory result (see [8] for the proof)

Lemma 1. Let $g(z, \lambda)=\frac{1}{1-\frac{\lambda}{\gamma}+\lambda e^{z}}$ for $z \in Z \equiv\left[\ln \left(\gamma^{-1}\right), \infty\right]$ and $\lambda \in \Lambda \equiv[0, \gamma]$ (recall $\gamma$ is our strictly positive upper bound for $\left.\gamma_{1}\right)$. Then, for any $q \geq 0,\left|D_{1}^{q} g(z, \lambda)\right| \leq 2^{q} q !, \quad \forall z \in Z, \forall \lambda \in \Lambda$, where $D_{1}^{q} g$ denotes the $q^{\text {th }}$-derivative of $g$ with respect to the first argument.

We now prove a bound for the best approximation result in

Lemma 2. For $N \geq N_{\text {crit }} \equiv c^{*} e \ln \left(\gamma \mu_{\max }+1\right)$

$$
\inf _{w_{N}^{\mathcal{N}} \in W_{N}^{N}}\left|\left\|u^{\mathcal{N}}(\mu)-w_{N}^{\mathcal{N}}\left|\|\leq\| u^{\mathcal{N}}(0)\right|\right\| \exp \left\{\frac{-N}{N_{\text {crit }}}\right\}, \quad \forall \mu \in \mathcal{D} .\right.
$$

Proof. To facilitate the proof, we shall effect a change of coordinates in parameter space. To wit, we let $\widetilde{\mathcal{D}} \equiv\left[\ln \gamma^{-1}, \ln \left(\mu_{\max }+\gamma^{-1}\right)\right]$, and introduce $\tau: \widetilde{\mathcal{D}} \rightarrow \mathcal{D}$ as $\tau(\tilde{\mu})=e^{\tilde{\mu}}-\gamma^{-1}$ so that $\tau^{-1}(\mu)=\ln \left(\mu+\gamma^{-1}\right)$. We then set $\tilde{u}(\tilde{\mu})=u(\tau(\tilde{\mu})), \tilde{u}^{\mathcal{N}}(\tilde{\mu})=u^{\mathcal{N}}(\tau(\tilde{\mu}))$, and $\tilde{u}_{N}^{\mathcal{N}}(\tilde{\mu})=u_{N}^{\mathcal{N}}(\tau(\tilde{\mu}))$. We note that

$$
\tilde{u}^{\mathcal{N}}(\tilde{\mu})=\sum_{i=1}^{\mathcal{N}} \frac{f_{i}^{\mathcal{N}} \varphi_{i}^{\mathcal{N}}}{1-\frac{\lambda_{i}^{\mathcal{N}}}{\gamma}+\lambda_{i}^{\mathcal{N}} e^{\tilde{\mu}}}=\sum_{i=1}^{\mathcal{N}} f_{i}^{\mathcal{N}} \varphi_{i}^{\mathcal{N}} g\left(\tilde{\mu}, \lambda_{i}^{\mathcal{N}}\right),
$$

from (6), our change of variable, and the definition of $g$.

We now observe that in our mapped coordinate, the sample points $\tilde{\alpha}_{k} \equiv \tau^{-1}\left(\alpha_{k}\right), k=1, \ldots, N$, are equi-distributed with separation $\tilde{\alpha}_{k+1}-\tilde{\alpha}_{k} \simeq \ln \left(\gamma \mu_{\max }+1\right) / N$. It thus follows that, given any $\tilde{\mu} \in \tilde{\mathcal{D}}$, we can construct a closed interval $\widetilde{I}_{\tilde{\Delta}}^{\tilde{\mu}}$ of length $\tilde{\Delta}$ that includes $\tilde{\mu}$ and $M^{\tilde{\mu}}\left(\tilde{\Delta}, \delta_{N}\right)$ distinct points $\tilde{\alpha}_{P_{n}^{\tilde{\mu}}}, n=1, \ldots, M$. Here $M^{\tilde{\mu}}\left(\tilde{\Delta}, \delta_{N}\right)$ is of the order of $\frac{\tilde{\Delta}}{\delta_{N}}$; more precisely,

$$
M^{\tilde{\mu}}\left(\tilde{\Delta}, \delta_{N}\right) \geq \frac{\tilde{\Delta}}{c^{*} \delta_{N}} .
$$


In what follows, we shall often abbreviate $M^{\tilde{\mu}}\left(\tilde{\Delta}, \delta_{N}\right)$ as $M$.

Now, for any $\tilde{\mu} \in \tilde{\mathcal{D}}$, we introduce $\hat{u}^{\tilde{\mu}} \in W_{N}^{\mathcal{N}}$ given by

$$
\hat{u}^{\tilde{\mu}} \equiv \sum_{n=1}^{M} \tilde{Q}_{n}^{\tilde{\mu}}(\tilde{\mu}) u^{\mathcal{N}}\left(\tau\left(\tilde{\alpha}_{P_{n}^{\tilde{\mu}}}\right)\right)=\sum_{n=1}^{M} \tilde{Q}_{n}^{\tilde{\mu}}(\tilde{\mu}) \tilde{u}^{\mathcal{N}}\left(\tilde{\alpha}_{P_{n}^{\tilde{\mu}}}\right)=\sum_{n=1}^{M} \tilde{Q}_{n}^{\tilde{\mu}}(\tilde{\mu}) \sum_{i=1}^{\mathcal{N}} f_{i}^{\mathcal{N}} \varphi_{i}^{\mathcal{N}} g\left(\tilde{\alpha}_{\left.P_{n}^{\tilde{\mu}}, \lambda_{i}^{\mathcal{N}}\right)},\right.
$$

where the characteristic functions $\widetilde{Q}_{n}^{\tilde{\mu}}$ are uniquely determined by $\widetilde{Q}_{n}^{\tilde{\mu}} \in \mathbb{P}_{M-1}\left(\tilde{I}_{\tilde{\Delta}}^{\tilde{\mu}}\right), n=1, \ldots, M$, and $\widetilde{Q}_{n}^{\tilde{\mu}}\left(\tilde{\alpha}_{P_{n^{\prime}}^{\tilde{\mu}}}\right)=\delta_{n n^{\prime}}, 1 \leq n, n^{\prime} \leq M$; here $\mathbb{P}_{M-1}\left(\tilde{I}_{\tilde{\Delta}}^{\tilde{\mu}}\right)$ refers to the space of polynomials of degree $\leq M-1$ over $\tilde{I}_{\tilde{\Delta}}^{\tilde{\mu}}$. We thus obtain

$$
\hat{u}^{\tilde{\mu}}=\sum_{i=1}^{\mathcal{N}} f_{i}^{\mathcal{N}} \varphi_{i}^{\mathcal{N}}\left[\tilde{\mathcal{I}}_{M-1}^{\tilde{\mu}} g\left(\cdot, \lambda_{i}^{\mathcal{N}}\right)\right](\tilde{\mu}),
$$

where, for given $\lambda, \widetilde{\mathcal{I}}_{M-1}^{\tilde{\mu}} g(\cdot, \lambda)$ is the $(M-1)^{\text {th }}$-order polynomial interpolant of $g(\cdot, \lambda)$ through the $\tilde{\alpha}_{P_{n}^{\tilde{\mu}}}, n=1, \ldots, M$; more precisely, $\tilde{\mathcal{I}}_{M-1}^{\tilde{\mu}} g(\cdot, \lambda) \in \mathbb{P}_{M-1}\left(\widetilde{I}_{\tilde{\Delta}}^{\tilde{\mu}}\right)$, and $\left(\widetilde{\mathcal{I}}_{M-1}^{\tilde{\mu}} g(\cdot, \lambda)\right)\left(\tilde{\alpha}_{P_{n}^{\tilde{\mu}}}\right)=$

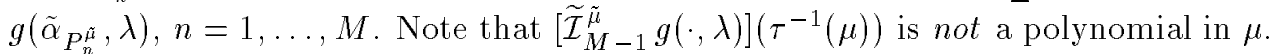

It now follows from (5), (6), (13) and (15) that

$$
\begin{aligned}
\left\|\tilde{u}^{\mathcal{N}}(\tilde{\mu})-\hat{u}^{\tilde{\mu}} \mid\right\| & \leq\left\|\mid \sum_{i=1}^{\mathcal{N}} f_{i}^{\mathcal{N}} \varphi_{i}^{\mathcal{N}}\left(g\left(\tilde{\mu}, \lambda_{i}^{\mathcal{N}}\right)-\left[\tilde{\mathcal{I}}_{M-1}^{\tilde{\mu}} g\left(\cdot, \lambda_{i}^{\mathcal{N}}\right)\right](\tilde{\mu})\right)\right\| \| \\
& \leq \sup _{\lambda \in \Lambda}\left|g(\tilde{\mu}, \lambda)-\left[\tilde{\mathcal{I}}_{M-1}^{\tilde{\mu}} g(\cdot, \lambda)\right](\tilde{\mu})\right|||\left|u^{\mathcal{N}}(0)\right| \| .
\end{aligned}
$$

We next invoke the standard polynomial interpolation remainder formula [3] and Lemma 1 to obtain

$$
\sup _{\lambda \in \Lambda}\left|g(\tilde{\mu}, \lambda)-\left[\widetilde{\mathcal{I}}_{M-1}^{\hat{u}} g(\cdot, \lambda)\right](\tilde{\mu})\right| \leq \sup _{\lambda \in \Lambda} \sup _{z \in Z} \frac{1}{M !}\left|D_{1}^{M} g(z, \lambda)\right| \tilde{\Delta}^{M} \leq(2 \tilde{\Delta})^{M^{\tilde{\mu}}\left(\tilde{\Delta}, \delta_{N}\right)} .
$$

We now assume that $\frac{c^{*} \delta_{N}}{2} \leq \tilde{\Delta}$ and $\tilde{\Delta} \leq \frac{1}{2}$; under these conditions (recall (14)) we obtain $(2 \tilde{\Delta})^{M^{\tilde{\mu}}\left(\tilde{\Delta}, \delta_{N}\right)} \leq(2 \tilde{\Delta})^{\tilde{\Delta} / c^{*} \delta_{N}}$, and hence, from (16) and (17), we can write

$$
\left\|\left|\tilde{u}^{\mathcal{N}}(\tilde{\mu})-\hat{u}^{\tilde{\mu}}\right|\right\| \leq\left\||| u^{\mathcal{N}}(0)\right\|||(2 \tilde{\Delta})^{\tilde{\Delta} / c^{*} \delta_{N}} .
$$

It remains to select a best $\tilde{\Delta}$ satisfying $\frac{c^{*} \delta_{N}}{2} \leq \tilde{\Delta} \leq \frac{1}{2}$.

To provide the sharpest possible bound, we choose $\tilde{\Delta}=\tilde{\Delta}^{*} \equiv \frac{1}{2 e}$, the minimizer (over all positive $\tilde{\Delta})$ of $(2 \tilde{\Delta})^{\tilde{\Delta} / \delta_{N}}$. Our conditions on $\tilde{\Delta}$ are readily verified: $\frac{c^{*} \delta_{N}}{2} \leq \tilde{\Delta}^{*}$ follows directly from the hypothesis of our lemma, $N \geq N_{\text {crit }}$; and $\tilde{\Delta}^{*} \leq \frac{1}{2}$ follows from inspection. We now insert $\tilde{\Delta}=\tilde{\Delta}^{*}$ into (18) to obtain $\left\|\left|\tilde{u}^{\mathcal{N}}(\tilde{\mu})-\hat{u}^{\tilde{\mu}}\right|\right\| \leq\left\|\mid u^{\mathcal{N}}(0)\right\| \| e^{-N / N_{\text {crit }}}$, for all $\tilde{\mu} \in \tilde{\mathcal{D}}$. It immediately follows that, for any $\mu \in \mathcal{D}$,

$$
\begin{array}{r}
\inf _{w_{N}^{N} \in W_{N}^{\mathcal{N}}}\left\|\left|u^{\mathcal{N}}(\mu)-w_{N}^{\mathcal{N}}\right|\right\|=\inf _{w_{N}^{\mathcal{N}} \in W_{N}^{N}}\left|\left\|\tilde{u}^{\mathcal{N}}\left(\tau^{-1}(\mu)\right)-w_{N}^{\mathcal{N}} \mid\right\|\right. \\
\leq\left\||| \tilde{u}^{\mathcal{N}}\left(\tau^{-1}(\mu)\right)-\hat{u}^{\tau^{-1}(\mu)}\left|\left\|\leq||\left|u^{\mathcal{N}}(0)\right|\right\| e^{-N / N_{\text {crit }}}\right.\right.
\end{array}
$$

since $\hat{u} \in W_{N}^{\mathcal{N}}$ and, for $\mu \in \mathcal{D}, \tau^{-1}(\mu) \in \tilde{\mathcal{D}}$. This concludes the proof.

Then, from (10),(11), Lemma 1, and Lemma 2, we obtain 
Theorem 3. For $N \geq N_{\text {crit }} \equiv c^{*} e \ln \left(\gamma \mu_{\max }+1\right)$,

$$
\left\|u^{\mathcal{N}}(\mu)-u_{N}^{\mathcal{N}}(\mu)\left|\left\|\leq\left(1+\mu_{\max } \gamma_{1}\right)^{1 / 2}\right\|\left\|u^{\mathcal{N}}(0)\right\|\right| e^{-N / N_{\text {crit }}}, \forall \mu \in \mathcal{D}\right.
$$

furthermore for $\mathcal{N}(\varepsilon)$ such that ||$\left|u(\mu)-u^{\mathcal{N}(\varepsilon)}(\mu)\right| \| \leq \varepsilon$,

$$
\left\|\left|u(\mu)-u_{N}^{\mathcal{N}(\varepsilon)}(\mu)\right||| \leq \varepsilon+\left(1+\mu_{\max } \gamma_{1}\right)\right\||| u(0) \mid \| e^{-N / N_{\text {crit }}}, \forall \mu \in \mathcal{D} .
$$

Remark 4. By letting $\varepsilon$ go to zero, we also have

$$
\left\|\left|u(\mu)-u_{N}(\mu)\||\leq c|\| u(0)\|\| e^{-N / N_{\text {crit }}}, \forall \mu \in \mathcal{D},\right.\right.
$$

for any $N \geq N_{\text {crit }}$ with a constant $c$ that depends only on $\gamma_{1}$ and $\mu_{\max }$.

Remark 5. It must be pointed out that the analysis of the best fit in lemma 2 involves a simple polynomial approximation of the solution, but this is a polynomial in the $\tilde{\mu}$ variable. The Galerkin approximation provides this best fit, up to a multiplicative constant, regardless of any a priori knowledge of the dependance of the solution on the parameter. This demonstrates the superiority of the reduced basis method with respect to a "simple" interpolation approximation.

Acknowledgements. We would like to thank Christophe Prud'homme, Dimitrios Rovas, and Karen Veroy of MIT for sharing their numerical results prior to publication. This work was performed while ATP was an Invited Professor at the University of Paris VI in February, 2001. This work was supported by the Singapore-MIT Alliance, by DARPA and ONR under Grant F49620-01-1-0458, by DARPA and AFOSR under Grant N00014-01-1-0523 (Subcontract 340-6218-3), and by NASA under Grant NAG-1-1978.

\section{References}

[1] Almroth B.O., Stern P., Brogan F.A., Automatic choice of global shape functions in structural analysis, AIAA Journal 16 (May 1978) 525-528.

[2] Barrett A., Reddien G., On the reduced basis method, Math. Mech. 7(75) (1995) 543-549.

[3] Dahlquist G., Björck Å., Numerical Methods, Prentice-Hall, 1974, p. 100.

[4] Fink J.P., Rheinboldt W.C., On the error behaviour of the reduced basis technique for nonlinear finite element approximations, Z. Angew. Math. Mech. 63 (1983) 21-28.

[5] Machiels L., Maday Y., Oliveira I.B., Patera A.T., Rovas D.V., Output bounds for reduced-basis approximations of symmetric positive definite eigenvalue problems, C. R. Acad. Sci. Paris, t. 331, Série I (2000) 153-158.

[6] Maday Y., Machiels L., Patera A.T., Rovas D.V., Blackbox reduced-basis output bound methods for shape optimization, Proceedings $12^{\text {th }}$ International Domain Decomposition Conference, Japan, 2000.

[7] Maday Y., Patera A.T., Rovas D.V., A blackbox reduced-basis output bound method for noncoercive linear problems. Nonlinear Partial Differential Equations and Their Applications, College De France Seminar, 2001.

[8] Maday Y., Patera A.T., Turinici G., A Priori Convergence Theory for Reduced-Basis Approximations of SingleParameter Elliptic Partial Differential Equations, in preparation.

[9] Noor A.K., Peters J.M., Reduced basis technique for nonlinear analysis of structures, AIAA Journal 18(4) (April 1980) 455-462.

[10] Peterson J.S., The reduced basis method for incompressible viscous flow calculations, SIAM J. Sci. Stat. Comput. 10(4) (July 1989) 777-786.

[12] Prud'homme C., Rovas, D.V., Veroy K., Machiels L., Maday Y., Patera A.T., Turinici G., Reliable Real-Time Solution of Parametrized Partial Differential Equations: Reduced-Basis Output Bound Methods, J. Fluids Engineering, submitted 2001.

[13] Rheinboldt W.C., On the theory and error estimation of the reduced basis method for multi-parameter problems, Nonlinear Analysis, Theory, Methods and Applications 21(11) (1993) 849-858.

[14] Strang W.G., Fix G.J., An Analysis of the Finite Element Method, Wellesley-Cambridge Press, 1973.

[15] Veroy K., Reduced Basis Methods Applied to Problems in Elasticity: Analysis and Applications, PhD thesis, Massachusetts Institute of Technology, 2003. In progress. 